\title{
Changes in coffee intake and subsequent risk of type 2 diabetes: three large cohorts of US men and women
}

\author{
Shilpa N. Bhupathiraju • An Pan • JoAnn E. Manson • \\ Walter C. Willett • Rob M. van Dam • Frank B. Hu
}

Received: 22 October 2013 / Accepted: 21 March 2014 / Published online: 26 April 2014

(C) Springer-Verlag Berlin Heidelberg 2014

\begin{abstract}
Aims/hypothesis Coffee and tea consumption has been associated with a lower type 2 diabetes risk but little is known about how changes in coffee and tea consumption influence subsequent type 2 diabetes risk. We examined the associations between 4 year changes in coffee and tea consumption and risk of type 2 diabetes in the subsequent 4 years.

Methods We prospectively followed 48,464 women in the Nurses' Health Study (NHS; 1986-2006), 47,510 women in NHS II (1991-2007) and 27,759 men in the Health Professionals Follow-up Study (HPFS; 1986-2006). Diet was assessed
\end{abstract}

Electronic supplementary material The online version of this article (doi:10.1007/s00125-014-3235-7) contains peer-reviewed but unedited supplementary material, which is available to authorised users.

S. N. Bhupathiraju • W. C. Willett • F. B. Hu $(\square)$

Department of Nutrition, Harvard School of Public Health,

655 Huntington Ave, Boston, MA 02115, USA

e-mail: nhbfh@channing.harvard.edu

A. Pan $\cdot$ R. M. van Dam

Saw Swee Hock School of Public Health and Yong Loo Lin School of Medicine, National University of Singapore and National

University Health System, Singapore, Republic of Singapore

J. E. Manson

Division of Preventive Medicine, Brigham and Women's Hospital

and Harvard Medical School, Boston, MA, USA

J. E. Manson • W. C. Willett • F. B. Hu

Department of Epidemiology, Harvard School of Public Health,

Boston, MA, USA

W. C. Willett $\cdot$ F. B. Hu

Channing Division of Network Medicine, Brigham and Women's

Hospital and Harvard Medical School, Boston, MA, USA every 4 years using a validated food-frequency questionnaire. Self-reported cases of incident type 2 diabetes were validated by supplementary questionnaires.

Results During 1,663,319 person-years of follow-up, we documented 7,269 cases of incident type 2 diabetes. Participants who increased their coffee consumption by more than $1 \mathrm{cup} /$ day (median change $=1.69$ cups/day) over a 4 year period had an $11 \%(95 \%$ CI $3 \%, 18 \%)$ lower risk of type 2 diabetes in the subsequent 4 years compared with those who made no changes in consumption. Participants who decreased their coffee intake by more than $1 \mathrm{cup} /$ day (median change $=-2$ cups/day) had a $17 \%(95 \% \mathrm{CI} 8 \%, 26 \%)$ higher risk for type 2 diabetes. Changes in tea consumption were not associated with type 2 diabetes risk.

Conclusions/interpretation Our data provide novel evidence that increasing coffee consumption over a 4 year period is associated with a lower risk of type 2 diabetes, while decreasing coffee consumption is associated with a higher risk of type 2 diabetes in subsequent years.

Keywords Caffeinated coffee $\cdot$ Change $\cdot$ Coffee $\cdot$ Decaffeinated coffee $\cdot$ Tea $\cdot$ Type 2 diabetes
Abbreviations
FFQ Food-frequency questionnaire
HPFS Health Professionals Follow-Up Study
NHS Nurses' Health Study

\section{Introduction}

Consumption of coffee and tea has consistently been associated with a lower risk for type 2 diabetes [1-3]. In a 
meta-analysis of 28 prospective studies, representing $1,109,272$ participants, every additional cup of caffeinated and decaffeinated coffee consumed in a day was associated with a $9 \%(95 \%$ CI 6\%, 11\%) and 6\% (95\% CI 2\%, 9\%) lower risk of type 2 diabetes, respectively [3]. In another metaanalysis, participants who drank more than three to four cups of tea per day had an $8 \%$ lower risk of type 2 diabetes [1]. However, because individuals frequently make changes to their diet, observational studies examining the association of baseline coffee consumption with type 2 diabetes risk cannot adequately capture these changes or account for secular trends in intake. Examining how changes in coffee and tea consumption affect risk of type 2 diabetes can provide a more complete understanding of the relationships between coffee, tea and type 2 diabetes. Further, evaluating the association of shortterm changes in coffee and tea intake with the occurrence of type 2 diabetes in subsequent years will help determine how quickly such dietary changes impact diabetes risk. To our knowledge, no study has examined the association between changes in coffee and tea consumption and risk of type 2 diabetes.

We used observational data from three large prospective studies, the Nurses' Health Study (NHS), the NHS II and the Health Professionals Follow-up Study (HPFS), to examine changes in coffee and tea intake in relation to risk of type 2 diabetes. In all three cohorts, we collected detailed information on diet, lifestyle, medical conditions and other chronic diseases every 2 to 4 years for over 20 years. The availability of these repeated measures and the long duration of follow-up allowed us to evaluate 4 year changes in coffee and tea intake in relation to risk of type 2 diabetes in the next 4 years. We also examined whether the association with diabetes incidence differed between changes in caffeinated and decaffeinated coffee intake. Finally, we evaluated the long-term associations of changes in coffee and tea intake by examining changes from baseline to the first 4 years of follow-up in relation to risk of type 2 diabetes in the subsequent 12 (in the NHS II) and 16 years (in the NHS and HPFS) of follow-up.

\section{Methods}

Study population The NHS was initiated in 1976 as a prospective cohort study of 121,701 female registered nurses, 30 55 years of age, from 11 US states. The NHS II consists of 116,681 younger female registered nurses, aged 25-42 years at baseline (1989). The HPFS is a prospective cohort study of 51,529 male health professionals, 40-75 years of age, from all 50 states, that began in 1986 . Cohort members received validated questionnaires at baseline and every 2 years thereafter to update their information on medical history, lifestyle, potential risk factors and disease diagnosis [4-8].
For the current investigation, we used 1986 for the NHS $(n=80,332)$ and HPFS $(n=38,842)$, and 1991 for the NHS II $(n=87,448)$, as our baseline when we first obtained detailed information on diet and lifestyle. Because our primary exposure was 4 year change in coffee and tea intake, we excluded participants with a history of diabetes (including type 1 diabetes, type 2 diabetes and gestational diabetes), cardiovascular disease or cancer 4 years after baseline (1990 for the NHS and HPFS and 1995 for the NHS II; $n=28,739$ ) because changes in diet after development of these conditions may confound the relationship between diet and disease [9]. We also excluded women who were pregnant during follow-up $(n=23,519)$. We excluded participants with $\geq 10$ food items blank, those with implausible energy intakes at baseline $(<3,347$ or $>17,573 \mathrm{~kJ} /$ day for men and $<2,092$ or $>14,644 \mathrm{~kJ} /$ day for women) and those participants who died before the baseline $(n=26,061)$. We also excluded participants with missing information on dietary and lifestyle covariables and those who returned only the baseline questionnaire $(n=4,570)$. The final study population consisted of 48,464 women in the NHS, 47,510 women in the NHS II and 27,759 men in the HPFS with complete information. The study was approved by the institutional review boards of Brigham and Women's Hospital and Harvard School of Public Health. All participants gave informed consent.

Ascertainment of diet In the NHS and HPFS, diet was assessed in 1986 using an 126 item semi-quantitative foodfrequency questionnaire (FFQ). In NHS II, diet was first assessed in 1991 using a similar FFQ. In all three cohorts, subsequent FFQs were administered every 4 years to update information on diet. In all FFQs, participants were asked how often, on average (from 'never' to ' $\geq 6$ times/day') during the previous year they had consumed caffeinated and decaffeinated coffee ('one cup') and tea ('one cup or glass'). Decaffeinated tea consumption was first ascertained in 1998 in NHS and HPFS and in 1995 in NHS II and was therefore not included in our analyses. The validity and reproducibility of the FFQs have been described in detail elsewhere [10-12]. In a validation study conducted among a subsample of the NHS participants, FFQ assessments of coffee and tea were highly correlated with diet record assessments (coffee, $r=0.78$; tea, $r=0.93$ ) [11]. Similar correlation coefficients were found in a validation study conducted in a subsample of the HPFS participants [13].

Ascertainment of type 2 diabetes The primary endpoint for this study was incident type 2 diabetes. Participants who reported a diagnosis of type 2 diabetes on the biennial main questionnaire were sent a supplementary questionnaire regarding symptoms, diagnostic tests and hypoglycaemic therapy. We used the National Diabetes Data Group criteria [14] for cases identified before 1998 and the American Diabetes 
Association criteria [15] for cases identified after 1998. The validity of the supplementary questionnaire has been established in two previous studies in the NHS and HPFS through medical record reviews. In both studies, diagnosis of type 2 diabetes was confirmed in more than $98 \%$ of the cases $[16,17]$.

Ascertainment of covariates In the biennial follow-up questionnaires, we updated information on a participant's age, weight, smoking status, physical activity, menopausal status and use of postmenopausal hormone therapy (for women), oral contraceptive use (for women) and personal history of chronic diseases. Height was ascertained at baseline in each cohort. The presence or absence of a family history of diabetes (in first-degree relatives) was assessed in: 1982 and 1988 in the NHS; 1989, 1997, 2001 and 2005 in the NHS II; and 1987 in the HPFS. Information on intake of alcohol and other beverages was updated every 4 years using the FFQ. We used the Alternate Healthy Eating Index 2010 as an overall measure of diet quality of study participants [18].

Statistical analysis We used changes in coffee and tea consumption that were updated every 4 years to predict risk of type 2 diabetes in the subsequent 4 years. Participants were divided into categories of: no change ( $\pm 1 \mathrm{cup} /$ week); small to moderate increase or decrease ( \pm 1 cup/week to 1 cup/day); and moderate to large increase or decrease in consumption $(>1$ cup/day). We calculated each individual's person-time from the return of the baseline questionnaire to the date of diagnosis of type 2 diabetes, death, date of loss to follow-up or the cutoff date (30 June 2006 in the NHS, 30 June 2007 in the NHS II and 1 January 2006 in the HPFS), whichever occurred first.

We used Cox proportional hazard models with timevarying covariates and age as the underlying time scale for all analyses. Our first multivariable model adjusted for several lifestyle factors, including race, family history of diabetes, menopausal status and postmenopausal hormone use (women only), oral contraceptive use (women only), history of hypertension and hypercholesterolaemia (updated every 2 years) and change in smoking status during the 4 year period (never to never, never to current, past to current, current to past, current to current, missing indicator). For each 4 year time period, we adjusted for baseline and 4 year changes in physical activity (quintiles), intake of alcohol (quintiles) and other beverages (including sugar-sweetened beverages, artificially sweetened beverages, punch; quantiles). Changes in coffee and tea consumption were mutually adjusted. Because those with poor or worsening health are more likely to visit their physician, we additionally adjusted for a history of physical examination in the last cycle. Because changes in caffeine intake were previously associated with weight gain [19] and to assess if BMI and weight change are potential mediators or confounders of the association between changes in coffee and tea intake and type 2 diabetes, we further included baseline BMI $(<21,21-22.9,23-24.9,25-26.9,27-29.9,30-34.9,35-$ 39.9 and $\geq 40 \mathrm{~kg} / \mathrm{m}^{2}$ ) and 4 year weight change (quintiles) in our multivariable-adjusted model.

We also examined the associations of 4 year changes in caffeinated and decaffeinated coffee, separately, on risk of type 2 diabetes. Caffeinated and decaffeinated coffee intakes were mutually adjusted. In sensitivity analyses, we examined the long-term effects of changes in coffee and tea intake on risk of type 2 diabetes. Specifically, we calculated changes in coffee and tea intake from baseline to the first 4 years of follow-up (1986 to 1990 in the NHS and HPFS; 1991 to 1995 in the NHS II) to predict risk of type 2 diabetes in the subsequent years of follow-up (1990 to 2006 in the NHS and HPFS; 1995 to 2007 in the NHS II). Because participants who are at risk for cardiovascular disease or cancer are more likely to make changes to their coffee and tea intake, in sensitivity analyses, we censored incident cases of cardiovascular disease and cancer during follow-up and then examined the association between 4 year changes in coffee and tea intake and risk of type 2 diabetes. We also tested for potential effect modification by current smoking status, presence of hypertension and hypercholesterolaemia, baseline BMI and changes in weight.

Because of differences in sex, follow-up time and questionnaires in the three cohorts, all analyses were performed separately in each cohort to achieve better control of confounding. For the primary analyses, to obtain overall estimates for both sexes and to increase statistical power, the HRs from the multivariable-adjusted models from the three cohorts were combined with the use of an inverse variance-weighted metaanalysis by the random-effects model which accounts for heterogeneity between studies [20]. All statistical tests were two-sided and performed using SAS version 9.2 for UNIX (SAS Institute, Cary, NC, USA).

\section{Results}

During 1,663,319 person-years of follow-up, we documented 7,269 incident cases of type 2 diabetes. Age-adjusted baseline characteristics according to categories of 4 year changes in total coffee consumption are presented in electronic supplementary material (ESM) Table 1. In all three cohorts, compared with those who had relatively stable coffee consumption patterns, those who made the largest decreases in coffee consumption had higher initial intakes of total coffee and alcohol, were more likely to be older and had greater weight gain over a 4 year period. In the HPFS and NHS II, these individuals also had higher initial intakes of total coffee and alcohol. Age-adjusted baseline characteristics according to categories of 4 year changes in total tea consumption are 
Table 1 HRs and 95\% CIs for incident type 2 diabetes according to updated 4 year changes in coffee and tea intake

\begin{tabular}{|c|c|c|c|c|c|c|}
\hline \multirow[t]{2}{*}{ Intake/study } & \multicolumn{5}{|c|}{ Changes in frequency of consumption } & \multirow[t]{2}{*}{ HR per 1 serving } \\
\hline & $\begin{array}{l}\text { Moderate to } \\
\text { large decrease } \\
(>1 \text { cup/day })\end{array}$ & $\begin{array}{l}\text { Small to moderate } \\
\text { decrease ( } 1 \text { cup/ } \\
\text { week-1 cup/day })\end{array}$ & $\begin{array}{l}\text { No change } \\
( \pm 1 \text { cup/week })^{\mathrm{a}}\end{array}$ & $\begin{array}{l}\text { Small to moderate } \\
\text { increase ( } 1 \text { cup/ } \\
\text { week-1 cup/day) }\end{array}$ & $\begin{array}{l}\text { Moderate to large } \\
\text { increase }(>1 \text { cup/day) }\end{array}$ & \\
\hline \multicolumn{7}{|l|}{ Coffee } \\
\hline \multicolumn{7}{|l|}{ HPFS } \\
\hline$n$ & 4,318 & 3,451 & 13,833 & 3,050 & 3,107 & \\
\hline Cases/person-years & $317 / 61,343$ & $199 / 49,540$ & $818 / 198,710$ & $182 / 44,571$ & $188 / 45,520$ & \\
\hline Multivariable adjusted $1^{\mathrm{b}}$ & $1.22(1.05,1.43)$ & $0.88(0.75,1.04)$ & 1.00 & $0.92(0.78,1.09)$ & $0.97(0.82,1.14)$ & $0.94(0.90,0.99)$ \\
\hline Multivariable adjusted $2^{\mathrm{c}}$ & $1.22(1.05,1.42)$ & $0.91(0.77,1.07)$ & 1.00 & $0.92(0.78,1.10)$ & $0.93(0.79,1.10)$ & $0.94(0.89,0.98)$ \\
\hline \multicolumn{7}{|l|}{ NHS } \\
\hline$n$ & 9,372 & 6,343 & 23,224 & 4,545 & 4,980 & \\
\hline Cases/person-years & $654 / 137,935$ & $449 / 93,692$ & $1,745 / 343,597$ & $343 / 67,890$ & $291 / 74,601$ & \\
\hline Multivariable adjusted $1^{\mathrm{b}}$ & $1.17(1.06,1.30)$ & $0.98(0.88,1.09)$ & 1.00 & $1.04(0.92,1.16)$ & $0.84(0.74,0.96)$ & $0.94(0.91,0.97)$ \\
\hline Multivariable adjusted $2^{\mathrm{c}}$ & $1.17(1.05,1.29)$ & $0.99(0.89,1.11)$ & 1.00 & $1.05(0.94,1.18)$ & $0.86(0.76,0.98)$ & $0.95(0.92,0.98)$ \\
\hline \multicolumn{7}{|l|}{ NHS II } \\
\hline$n$ & 7,132 & 5,214 & 25,536 & 4,786 & 4,841 & \\
\hline Cases/person-years & $278 / 80,074$ & $236 / 59,562$ & $1,201 / 293,809$ & $195 / 55,872$ & $173 / 56,604$ & \\
\hline Multivariable adjusted $1^{\mathrm{b}}$ & $1.16(0.99,1.36)$ & $0.96(0.82,1.13)$ & 1.00 & $0.86(0.73,1.01)$ & $0.86(0.73,1.02)$ & $0.94(0.90,0.99)$ \\
\hline Multivariable adjusted $2^{\mathrm{c}}$ & $1.10(0.94,1.30)$ & $0.94(0.80,1.11)$ & 1.00 & $0.90(0.76,1.06)$ & $0.90(0.76,1.07)$ & $0.97(0.92,1.01)$ \\
\hline \multicolumn{7}{|l|}{ Pooled $^{\mathrm{d}}$} \\
\hline Multivariable adjusted $1^{\mathrm{b}}$ & $1.18(1.10,1.28)$ & $0.95(0.88,1.03)$ & 1.00 & $0.95(0.84,1.06)$ & $0.88(0.81,0.96)$ & $0.94(0.92,0.96)$ \\
\hline Multivariable adjusted $2^{\mathrm{c}}$ & $1.17(1.08,1.26)$ & $0.96(0.89,1.04)$ & 1.00 & $0.97(0.87,1.08)$ & $0.89(0.82,0.97)$ & $0.95(0.93,0.97)$ \\
\hline \multicolumn{7}{|l|}{ Tea } \\
\hline \multicolumn{7}{|l|}{ HPFS } \\
\hline$n$ & 1,375 & 3,510 & 18,735 & 3,016 & 1,123 & \\
\hline Cases/person-years & $84 / 19,634$ & $229 / 50,421$ & $1,125 / 269,457$ & $187 / 43,859$ & $79 / 16,313$ & \\
\hline Multivariable adjusted $1^{\mathrm{b}}$ & $1.00(0.78,1.30)$ & $1.10(0.91,1.33)$ & 1.00 & $1.03(0.88,1.21)$ & $1.20(0.94,1.53)$ & $1.02(0.95,1.10)$ \\
\hline Multivariable adjusted $2^{\mathrm{c}}$ & $0.94(0.73,1.22)$ & $1.10(0.91,1.32)$ & 1.00 & $1.03(0.88,1.21)$ & $1.15(0.90,1.46)$ & $1.02(0.95,1.10)$ \\
\hline \multicolumn{7}{|l|}{ NHS } \\
\hline$n$ & 3,467 & 7,159 & 29,011 & 5,991 & 2,836 & \\
\hline Cases/person-years & $256 / 51,164$ & $548 / 105,883$ & $2,076 / 429,649$ & $395 / 88,888$ & $207 / 42,131$ & \\
\hline Multivariable adjusted $1^{\mathrm{b}}$ & $1.06(0.90,1.25)$ & $1.04(0.92,1.17)$ & 1.00 & $0.90(0.81,1.01)$ & $1.06(0.91,1.23)$ & $0.98(0.94,1.02)$ \\
\hline Multivariable adjusted $2^{\mathrm{c}}$ & $1.02(0.87,1.20)$ & $1.00(0.89,1.12)$ & 1.00 & $0.90(0.80,1.00)$ & $1.01(0.87,1.18)$ & $0.98(0.94,1.02)$ \\
\hline \multicolumn{7}{|l|}{ NHS II } \\
\hline$n$ & 4,530 & 8,953 & 23,211 & 7,440 & 3,376 & \\
\hline Cases/person-years & $223 / 51,672$ & $432 / 102,393$ & $957 / 266,493$ & $325 / 86,328$ & $146 / 39,034$ & \\
\hline Multivariable adjusted $1^{\mathrm{b}}$ & $1.18(0.96,1.44)$ & $1.19(1.03,1.37)$ & 1.00 & $1.05(0.92,1.20)$ & $0.95(0.79,1.15)$ & $0.96(0.91,1.00)$ \\
\hline Multivariable adjusted $2^{\mathrm{c}}$ & $1.12(0.92,1.37)$ & $1.18(1.02,1.36)$ & 1.00 & $1.05(0.92,1.20)$ & $0.94(0.78,1.13)$ & $0.96(0.92,1.00)$ \\
\hline \multicolumn{7}{|l|}{ Pooled $^{\mathrm{d}}$} \\
\hline Multivariable adjusted $1^{\mathrm{b}}$ & $1.08(0.97,1.22)$ & $1.10(1.01,1.19)$ & 1.00 & $0.98(0.89,1.09)$ & $1.05(0.94,1.17)$ & $0.98(0.95,1.01)$ \\
\hline Multivariable adjusted $2^{\mathrm{c}}$ & $1.03(0.92,1.16)$ & $1.08(0.97,1.20)$ & 1.00 & $0.98(0.88,1.09)$ & $1.01(0.91,1.12)$ & $0.98(0.95,1.01)$ \\
\hline
\end{tabular}

\footnotetext{
${ }^{a}$ Reference category

${ }^{\mathrm{b}}$ Adjusted for age, family history of diabetes (yes/no), race (white/non-white), postmenopausal hormone use (premenopausal, postmenopausal current user, postmenopausal never/past user, missing), physical examination in the previous cycle (yes/no), change in smoking status (never to never, never to current, current to current, current to past, past to current, past to past), baseline alcohol intake (0, 0.1-4.9, 5-9.9, 10-14.9, $\geq 15 \mathrm{~g} / \mathrm{day})$, change in alcohol intake (quintiles), baseline physical activity $(<3,3-8.9,9-17.9,18-26.9, \geq 27$ metabolic equivalents of task (METS)/week), change in physical activity (quintiles), history of hypertension and hypercholesterolaemia (yes/no), baseline and 4 year changes in beverage intake (quantiles; beverages include sugar-sweetened beverages, artificially sweetened beverages, punch, milk, coffee/tea depending on the model), baseline Alternate Healthy Eating Index score, and change in Alternate Healthy Eating Index score

${ }^{\mathrm{c}}$ Multivariable model $1+$ baseline BMI (<21, 21-22.9, 23-24.9, 25-26.9, 27-29.9, 30-34.9, 35-39.9, $\left.\geq 40 \mathrm{~kg} / \mathrm{m}^{2}\right)$ and change in body weight (quintiles)

${ }^{\mathrm{d}}$ The results across the three cohorts were pooled using an inverse variance-weighted random-effects meta-analysis
} 
shown in ESM Table 2. In all three cohorts, participants who changed their tea consumption the most had higher baseline tea consumption. In the NHS and NHS II, these participants also had lower initial intakes of total coffee. The Pearson correlation coefficient for total coffee intake between two adjacent FFQ years was $\sim 0.7(p<0.0001)$ in all three cohorts. For total tea, the correlation was $\sim 0.6(p<0.0001)$ in the NHS, NHS II and HPFS.

The HRs and 95\% CIs for type 2 diabetes associated with 4 year changes in coffee and tea consumption are presented in Table 1. Compared with those who made no changes to their coffee consumption, participants who had moderate to large increases in intake ( $>1$ cup/day) over a 4 year period had a $12 \%$ lower risk $(95 \%$ CI $4 \%, 19 \%)$ for type 2 diabetes in the next 4 years. Further adjustment for baseline BMI and concurrent weight change only slightly attenuated these results (11\% lower risk; $95 \%$ CI 3\%, 18\%). In pooled analyses, for those with moderate to large decreases in coffee consumption ( $>1$ cup/day) there was a $17 \%$ higher risk $(95 \%$ CI $8 \%, 26 \%)$ for type 2 diabetes. There was no association between updated 4 year changes in tea intake and subsequent risk for type 2 diabetes (Table 1).

Table 2 shows the HR for type 2 diabetes by change in different type of coffee consumption. Participants who had moderate to large increases in caffeinated coffee consumption ( $>1 \mathrm{cup} /$ day) had a $13 \%$ lower risk $(95 \%$ CI $5 \%$, $21 \%$ ) for type 2 diabetes compared with those who had relatively stable caffeinated coffee consumption over a 4 year period. Furthermore, those who had moderate to large decreases in their caffeinated coffee consumption had a $20 \%$ higher risk $(95 \%$ CI $10 \%, 30 \%)$ for type 2 diabetes in the subsequent 4 years. Changes in decaffeinated coffee consumption were not associated with risk for type 2 diabetes (Table 2).

The HRs for type 2 diabetes by initial and 4 year coffee consumption categories are presented in Fig. 1. Compared with those who were stable low consumers $(<1$ cup/day at baseline and 4 years later, reference group), those who were low consumers but increased their coffee consumption to more than 3 cups per day had a non-significantly lower risk for type 2 diabetes (HR $0.77 ; 95 \%$ CI $0.55,1.08$ ). The high-stable consumers had the lowest risk, a $37 \%$ lower risk $(95 \%$ CI $-44 \%,-28 \%$ ) compared with the low-stable consumers. No significant associations were observed for tea consumption categories and risk of type 2 diabetes. We found no statistically significant effect modification by current smoking status, presence of hypertension and hypercholesterolaemia, baseline BMI, and changes in weight ( $p$ for interaction $>0.05$ ).

To examine the long-term associations of changes in coffee and tea intake on type 2 diabetes risk, we used changes in coffee and tea consumption from baseline to the first 4 years of follow-up to predict risk for type 2 diabetes in the subsequent 12 years (in the NHS II) and 16 years (in the NHS and HPFS) of follow-up (ESM Table 3). In pooled multivariable analyses, participants who had moderate to large increases in total coffee consumption had a $13 \%$ lower risk $(95 \%$ CI $5 \%$, $21 \%$ ) for type 2 diabetes compared with those who had relatively stable intakes. Conversely, decreasing coffee consumption by more than 1 cup per day was not associated with an increased risk for type 2 diabetes (HR 1.09; 95\% CI 0.92, 1.30).

In the sensitivity analysis, we censored participants diagnosed with cardiovascular disease or cancer during follow-up (ESM Table 4). Participants with moderate to large increases in coffee consumption had an $11 \%$ lower risk $(95 \%$ CI $3 \%$, $19 \%$ ) for type 2 diabetes compared with stable drinkers. Furthermore, those who had moderate to large decreases in intake had an 18\% higher risk (95\% CI 9\%, 28\%). Similar to our primary analysis, there were no significant associations with changes in tea intake.

\section{Discussion}

In these three large prospective cohorts with more than 1.6 million person-years of follow-up, we observed that increasing intake of coffee, but not tea, over a 4 year period was associated with a lower type 2 diabetes risk in the next 4 years. Decreasing coffee intake was associated with a higher risk of type 2 diabetes. These changes in risk were observed for caffeinated, but not decaffeinated, coffee and were independent of initial coffee consumption and 4 year changes in other dietary and lifestyle factors.

A previous meta-analysis of prospective cohort studies has confirmed a clear inverse relation between coffee consumption and risk of type 2 diabetes; a $7 \%$ risk reduction was seen for every additional cup of coffee consumed per day [1]. In addition, our most recent findings from the NHS and HPFS also support a protective role of both caffeinated and decaffeinated coffee against the development of type 2 diabetes [21]. The findings of the current study not only confirm these previous reports but also demonstrate that change in coffee consumption is associated with both immediate and long-term diabetes risk. While randomised controlled trials are ideal to address the true causal relationship between changes in coffee and risk of type 2 diabetes, such studies are difficult to conduct given the long follow-up time needed for the development of type 2 diabetes, high costs, uncertainty regarding the ideal intervention period and the possibility of large attrition in adherence to the assigned beverage. Our study design can, to some extent, address these methodological difficulties by taking advantage of the large 
Table 2 HRs and 95\% CIs for incident type 2 diabetes according to updated 4 year changes in caffeinated and decaffeinated coffee intake

\begin{tabular}{|c|c|c|c|c|c|c|}
\hline \multirow[t]{2}{*}{ Intake/study } & \multicolumn{5}{|c|}{ Changes in frequency of coffee consumption } & \\
\hline & $\begin{array}{l}\text { Moderate to } \\
\text { large decrease } \\
(>1 \text { cup/day })\end{array}$ & $\begin{array}{l}\text { Small to moderate } \\
\text { decrease ( } 1 \text { cup/ } \\
\text { week-1 cup/day) }\end{array}$ & $\begin{array}{l}\text { No change } \\
( \pm 1 \text { cup/week })^{\mathrm{a}}\end{array}$ & $\begin{array}{l}\text { Small to moderate } \\
\text { increase ( } 1 \text { cup/ } \\
\text { week-1 cup/day) }\end{array}$ & $\begin{array}{l}\text { Moderate to large } \\
\text { increase }(>1 \text { cup/day) }\end{array}$ & \\
\hline \multicolumn{7}{|l|}{ Caffeinated coffee } \\
\hline \multicolumn{7}{|l|}{ HPFS } \\
\hline$n$ & 3,515 & 2,141 & 17,118 & 2,191 & 2,794 & \\
\hline Cases/person-years & $260 / 50,252$ & $141 / 30,340$ & $1,005 / 246,171$ & $138 / 31,963$ & $160 / 40,959$ & \\
\hline Multivariable adjusted $1^{\mathrm{b}}$ & $1.29(1.09,1.54)$ & $1.05(0.85,1.30)$ & 1.00 & $1.05(0.86,1.26)$ & $0.97(0.81,1.16)$ & $0.93(0.89,0.98)$ \\
\hline Multivariable adjusted $2^{c}$ & $1.28(1.08,1.53)$ & $1.01(0.82,1.26)$ & 1.00 & $1.02(0.84,1.23)$ & $0.91(0.76,1.10)$ & $0.92(0.88,0.97)$ \\
\hline \multicolumn{7}{|l|}{ NHS } \\
\hline$n$ & 7,600 & 3,394 & 28,872 & 2,901 & 4,596 & \\
\hline Cases/person-years & $512 / 112,119$ & $276 / 49,568$ & $2,190 / 443,893$ & $261 / 43,205$ & $243 / 68,931$ & \\
\hline Multivariable adjusted $1^{\mathrm{b}}$ & $1.18(1.05,1.33)$ & $1.02(0.88,1.18)$ & 1.00 & $1.17(1.02,1.34)$ & $0.81(0.70,0.93)$ & $0.94(0.91,0.98)$ \\
\hline Multivariable adjusted $2^{c}$ & $1.18(1.04,1.33)$ & $0.99(0.85,1.14)$ & 1.00 & $1.14(1.00,1.31)$ & $0.81(0.70,0.93)$ & $0.95(0.91,0.98)$ \\
\hline \multicolumn{7}{|l|}{ NHS II } \\
\hline$n$ & 6,421 & 3,196 & 29,873 & 3,425 & 4,595 & \\
\hline Cases/person-years & $243 / 72,453$ & $180 / 35,709$ & $1,342 / 344,074$ & $155 / 39,889$ & $163 / 53,795$ & \\
\hline Multivariable adjusted $1^{\mathrm{b}}$ & $1.21(1.02,1.44)$ & $1.02(0.84,1.23)$ & 1.00 & $1.01(0.85,1.21)$ & $0.91(0.76,1.09)$ & $0.95(0.91,1.00)$ \\
\hline Multivariable adjusted $2^{\mathrm{c}}$ & $1.15(0.97,1.38)$ & $0.97(0.80,1.18)$ & 1.00 & $1.01(0.84,1.20)$ & $0.93(0.78,1.11)$ & $0.97(0.92,1.02)$ \\
\hline \multicolumn{7}{|c|}{ Pooled $^{\mathrm{d}}$} \\
\hline Multivariable adjusted $1^{\mathrm{b}}$ & $1.21(1.11,1.32)$ & $1.02(0.92,1.14)$ & 1.00 & $1.09(0.99,1.20)$ & $0.88(0.79,0.98)$ & $0.94(0.92,0.97)$ \\
\hline Multivariable adjusted $2^{\mathrm{c}}$ & $1.20(1.10,1.30)$ & $0.99(0.89,1.09)$ & 1.00 & $1.07(0.97,1.18)$ & $0.87(0.79,0.95)$ & $0.95(0.92,0.97)$ \\
\hline \multicolumn{7}{|l|}{ Decaffeinated coffee } \\
\hline \multicolumn{7}{|l|}{ HPFS } \\
\hline$n$ & 1,925 & 2,794 & 19,307 & 2,282 & 1,451 & \\
\hline Cases/person-years & $129 / 27,687$ & $150 / 40,236$ & $1,193 / 277,172$ & $138 / 33,328$ & $94 / 21,261$ & \\
\hline Multivariable adjusted $1^{\mathrm{b}}$ & $1.16(0.93,1.45)$ & $0.99(0.80,1.22)$ & 1.00 & $0.91(0.76,1.09)$ & $0.97(0.78,1.21)$ & $0.96(0.90,1.03)$ \\
\hline Multivariable adjusted $2^{c}$ & $1.12(0.90,1.40)$ & $1.01(0.82,1.25)$ & 1.00 & $0.95(0.79,1.15)$ & $0.96(0.77,1.21)$ & $0.96(0.90,1.03)$ \\
\hline \multicolumn{7}{|l|}{ NHS } \\
\hline$n$ & 4,360 & 5,020 & 32,313 & 3,730 & 3,042 & \\
\hline Cases/person-years & $272 / 64,830$ & $345 / 74,308$ & $2,420 / 477,442$ & $254 / 55,573$ & $191 / 45,563$ & \\
\hline Multivariable adjusted $1^{\mathrm{b}}$ & $0.98(0.84,1.13)$ & $1.04(0.91,1.19)$ & 1.00 & $0.90(0.79,1.03)$ & $0.93(0.80,1.09)$ & $0.95(0.91,0.99)$ \\
\hline Multivariable adjusted $2^{\mathrm{c}}$ & $0.96(0.82,1.11)$ & $1.02(0.90,1.17)$ & 1.00 & $0.94(0.82,1.07)$ & $0.95(0.82,1.11)$ & $0.97(0.92,1.01)$ \\
\hline \multicolumn{7}{|l|}{ NHS II } \\
\hline$n$ & 2,134 & 4,721 & 35,296 & 3,642 & 1,717 & \\
\hline Cases/person-years & $63 / 24,596$ & $180 / 54,079$ & $1,666 / 404,713$ & $121 / 42,485$ & $53 / 20,048$ & \\
\hline Multivariable adjusted $1^{\mathrm{b}}$ & $1.25(0.85,1.85)$ & $0.93(0.79,1.10)$ & 1.00 & $0.71(0.59,0.86)$ & $0.75(0.56,0.99)$ & $0.89(0.81,0.97)$ \\
\hline Multivariable adjusted $2^{c}$ & $1.19(0.81,1.76)$ & $0.98(0.82,1.15)$ & 1.00 & $0.78(0.65,0.95)$ & $0.88(0.67,1.17)$ & $0.94(0.86,1.03)$ \\
\hline \multicolumn{7}{|l|}{ Pooled $^{\mathrm{d}}$} \\
\hline Multivariable adjusted $1^{\mathrm{b}}$ & $1.07(0.92,1.23)$ & $0.99(0.91,1.09)$ & 1.00 & $0.84(0.72,0.98)$ & $0.90(0.80,1.02)$ & $0.94(0.91,0.98)$ \\
\hline Multivariable adjusted $2^{\mathrm{c}}$ & $1.02(0.91,1.15)$ & $1.01(0.92,1.10)$ & 1.00 & $0.90(0.80,1.00)$ & $0.94(0.84,1.06)$ & $0.96(0.93,1.00)$ \\
\hline
\end{tabular}

\footnotetext{
${ }^{a}$ Reference category

${ }^{\mathrm{b}}$ Adjusted for age, family history of diabetes (yes/no), race (white/non-white), postmenopausal hormone use (premenopausal, postmenopausal current user, postmenopausal never/past user, missing), physical examination in the previous cycle (yes/no), change in smoking status (never to never, never to current, current to current, current to past, past to current, past to past) baseline alcohol intake $(0,0.1-4.9,5-9.9,10-14.9, \geq 15 \mathrm{~g} / \mathrm{day})$, change in alcohol intake (quintiles), baseline physical activity $(<3,3-8.9,9-17.9,18-26.9, \geq 27$ metabolic equivalents of task (METS)/week), change in physical activity (quintiles), history of hypertension and hypercholesterolaemia (yes/no), baseline and 4 year changes in beverage intake (quintiles or tertiles; beverages include sugar-sweetened beverages, artificially sweetened beverages, punch, milk, tea, caffeinated coffee/decaffeinated coffee depending on the model), baseline Alternate Healthy Eating Index score, and change in Alternate Healthy Eating Index score

${ }^{\mathrm{c}}$ Multivariable model $1+$ baseline BMI (<21, 21-22.9, 23-24.9, 25-26.9, 27-29.9, 30-34.9, 35-39.9, $\left.\geq 40 \mathrm{~kg} / \mathrm{m}^{2}\right)$ and change in body weight (quintiles)

${ }^{\mathrm{d}}$ The results across the three cohorts were pooled using an inverse variance-weighted random-effects meta-analysis
} 
$\mathbf{a}$

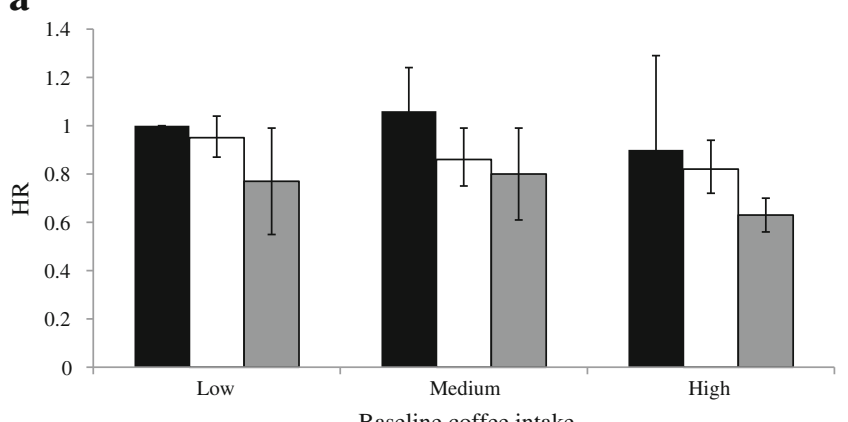

Baseline coffee intake

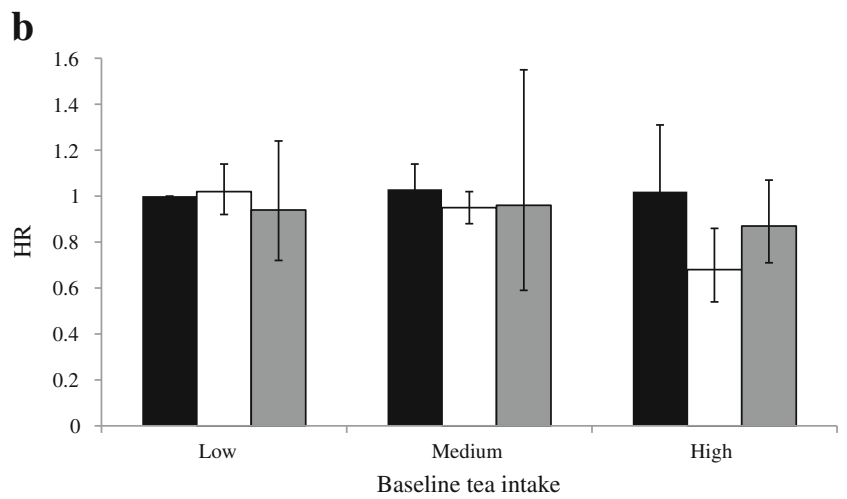

Fig. 1 HRs (and 95\% CIs) for type 2 diabetes according to updated 4 year changes in intake of (a) coffee and (b) tea. Low intake was defined as $<1$ cup per day; medium intake, 1-3 cups per day; and high intake, $>3$ cups per day. The vertical bars represent coffee or tea consumption 4 years later; black bars, low intake; white bars, medium intake; and grey bars, high intake ( $>3$ cups per day). The reference group (HR 1.00) was the low intake level at both baseline and the 4 year follow-up visits. The results across the three cohorts were pooled using an inverse variance-weighted random-effects meta-analysis

follow-up period and the availability of repeated measures of diet in our cohorts.

The higher risk of type 2 diabetes associated with decreasing coffee intake may represent a true change in risk or may potentially reflect reverse causation, whereby those with medical conditions associated with risk for type 2 diabetes (e.g. hypertension, elevated cholesterol, cardiovascular disease, cancer) may reduce their coffee consumption after diagnosis. However, when we censored participants who developed cardiovascular disease or cancer during follow-up, our results remained largely unchanged. Further, adjustment for hypertension and high cholesterol in our statistical models did not attenuate the association. When we examined the changes in the different types of coffee in relation to type 2 diabetes risk, only changes in caffeinated coffee consumption were associated with a reduced type 2 diabetes risk. Given the low consumption of decaffeinated coffee and the small proportion of participants who made substantial changes to their decaffeinated coffee consumption habits, the power to detect associations with changes in decaffeinated coffee could be limited. However, similar to our previous findings [21], we observed that initial decaffeinated coffee was independently associated with a reduced risk for type 2 diabetes (data not shown).

Our study showed that the association between increasing coffee consumption and a lower risk of type 2 diabetes in the short term ( 4 years) persists in the long term. When we used baseline 4 year changes in coffee intake to predict risk for type 2 diabetes in the entire follow-up, the risk reduction in type 2 diabetes associated with increasing coffee consumption (HR, $0.87 ; 95 \%$ CI $0.79,0.95$ ) was similar to that in our primary analysis (HR 0.89; 95\% CI 0.82, 0.97). However, unlike in our primary analysis, we did not document a higher risk of type 2 diabetes incidence with decreases in coffee intake, possibly because the analysis using baseline changes is less prone to reverse causation. Further, because this analysis does not take into account changes in coffee intake during the 4 year follow-up intervals, it is possible that those who increased their coffee consumption during the first 4 years had stable higher intakes in the subsequent years. On the other hand, it may be that decreases in coffee consumption in the first 4 years were more temporary and the associations were attenuated by subsequent increases in intake during follow-up. Our joint analysis of initial and coffee consumption 4 years later demonstrates that regardless of initial coffee consumption, increasing intake over 4 years is associated with a lower risk for type 2 diabetes. As expected, those who maintained high intakes at both baseline and 4 years later had the lowest risk for type 2 diabetes. For participants who changed from high to moderate levels, the protective association was maintained but was somewhat lower compared with those who had consistently high intakes.

Our data are largely consistent with findings from a 16 week randomised trial in which both caffeinated and decaffeinated coffee consumption was associated with modest decreases in post-load glucose levels [22]. In an 8 week intervention study, coffee consumption increased levels of adiponectin, an insulin-sensitising adipokine, but did not change measures of glycaemia or insulin sensitivity [23]. Our findings confirm results from a recent meta-analysis of prospective studies that documented $9 \%$ and $6 \%$ risk reductions for each additional cup of caffeinated and decaffeinated coffee, respectively [3]. In our change analysis, each additional cup of coffee was associated with a 5\% lower risk of type 2 diabetes. Although the meta-analysis by Huxley et al [1] reported an inverse association between tea and type 2 diabetes risk, we found no evidence of an association between 4 year increase in tea consumption and subsequent risk of type 2 diabetes. This finding may have been due to the relatively low number of participants who made significant changes to their tea consumption over a 4 year period, thereby limiting the statistical power to detect true associations. The overall low levels of tea consumption in this group may also be responsible for these findings. Our inability to differentiate between the various types of tea (green, black, other types) 
which have differential associations with type 2 diabetes risk [24] may have caused non-differential misclassification, biasing our results toward the null. Alternatively, it may be that changes in tea consumption are not associated with type 2 diabetes risk.

Our study has several strengths. The large sample size, the long follow-up period and presence of repeated dietary and covariable data are unique strengths in our study. Given the availability of repeated dietary information, we were able to assess the associations of dynamic changes in coffee and tea intake, independent of initial intake, with type 2 diabetes risk. However, our results need to be interpreted in the context of a few limitations. First, the participants in our study may be dissimilar to those in the general population because our study populations consisted of health professionals of primarily European ancestry. However, the high educational status of our study participants can be advantageous because reliable and valid dietary and questionnaire data can be captured [25]. Moreover, biological effects of coffee consumption on the development of type 2 diabetes are likely to be similar across socioeconomic groups and the results of our coffee-change analysis are consistent with results for initial coffee consumption and diabetes risk in a meta-analysis of cohorts including a wide variety of populations. Second, although we were able to carefully adjust for known dietary and lifestyle risk factors, residual confounding is of concern. For example, although sleep and depression are confounders of the association between changes in beverage intake and type 2 diabetes, these factors were not measured at all cycles. Further, while we adjusted for hypertension, other unmeasured potential illnesses that can change beverage consumption can confound the association between changes in coffee and tea intake and subsequent risk of type 2 diabetes. Third, dietary information was self-reported and assessed by FFQs. Therefore, some measurement error and misclassification is inevitable. However, the FFQs used in these studies were validated against multiple diet records $[10,13]$. Further, any measurement error is likely to be non-differential and therefore will attenuate associations toward the null. Fourth, because diet was assessed only every 4 years, we do not know when during the 4 year period participants made changes to their coffee and tea intake.

In conclusion, in this large study of US men and women, we found that increasing coffee consumption was associated with a lower type 2 diabetes risk. On the other hand, decreasing coffee consumption by the same amount was associated with higher diabetes risk in subsequent years. Changes in coffee consumption habit appear to affect diabetes risk in a relatively short amount of time. Our findings confirm those of prospective studies that higher coffee consumption is associated with a lower type 2 diabetes risk, and provide novel evidence that changes in coffee consumption habit is related to diabetes risk.
Acknowledgements We are indebted to the participants in the NHS, NHS II and HPFS for their continuing outstanding support and colleagues working in these studies for their valuable help.

Funding This study was funded by research grants P01 CA87969, P01 CA055075, R01 HL034594 and HL60712 from the National Institutes of Health. The work of SNB was supported by a postdoctoral fellowship grant from the American Heart Association (grant number 13POST14370012).

Duality of interest RMvD received grant funding from Nestec for a randomised trial of the effects of coffee consumption on insulin sensitivity. Nestec is a broad food company that also sells coffee. This is grant funding specific for that project with a contractual agreement that ensures that the company cannot influence the design of the study or decision to publish the results. This funding does not in any way affect the current study. Other authors declare that there is no duality of interest associated with this manuscript.

Contribution statement SNB analysed the data and drafted the paper. $\mathrm{AP}, \mathrm{WCW}, \mathrm{JEM}, \mathrm{RMvD}$ and $\mathrm{FBH}$ contributed to the conception and design of the study and acquisition of the data. All authors contributed to the interpretation of data and critical revision of the manuscript and approved the final version. SNB and FBH share primary responsibility for the final content.

\section{References}

1. Huxley R, Lee CM, Barzi F et al (2009) Coffee, decaffeinated coffee, and tea consumption in relation to incident type 2 diabetes mellitus: a systematic review with meta-analysis. Arch Intern Med 169:2053-2063

2. van Dam RM, Hu FB (2005) Coffee consumption and risk of type 2 diabetes: a systematic review. JAMA 294:97-104

3. Ding M, Bhupathiraju SN, Chen M, van Dam RM, Hu FB (2014) Caffeinated and decaffeinated coffee consumption and risk of type 2 diabetes: a systematic review and a dose-response meta-analysis. Diabetes Care 37:569-586

4. Belanger C, Speizer FE, Hennekens CH, Rosner B, Willett W, Bain C (1980) The nurses' health study: current findings. Am J Nurs 80:1333

5. Belanger CF, Hennekens CH, Rosner B, Speizer FE (1978) The Nurses' Health Study. Am J Nurs 78:1039-1040

6. Colditz GA, Manson JE, Hankinson SE (1997) The Nurses' Health Study: 20-year contribution to the understanding of health among women. J Women's Health 6:49-62

7. Mozaffarian D, Hao T, Rimm EB, Willett WC, Hu FB (2011) Changes in diet and lifestyle and long-term weight gain in women and men. N Engl J Med 364:2392-2404

8. Rimm EB, Giovannucci EL, Willett WC et al (1991) Prospective study of alcohol consumption and risk of coronary disease in men. Lancet 338:464-468

9. Hu FB, Stampfer MJ, Rimm E et al (1999) Dietary fat and coronary heart disease: a comparison of approaches for adjusting for total energy intake and modeling repeated dietary measurements. Am J Epidemiol 149:531-540

10. Rimm EB, Giovannucci EL, Stampfer MJ, Colditz GA, Litin LB, Willett WC (1992) Reproducibility and validity of an expanded selfadministered semiquantitative food frequency questionnaire among male health professionals. Am J Epidemiol 135:1114-1126, discussion $1127-1136$

11. Salvini S, Hunter DJ, Sampson L et al (1989) Food-based validation of a dietary questionnaire: the effects of week-to-week variation in food consumption. Int J Epidemiol 18:858-867 
12. Willett WC, Sampson L, Stampfer MJ et al (1985) Reproducibility and validity of a semiquantitative food frequency questionnaire. Am J Epidemiol 122:51-65

13. Feskanich D, Rimm EB, Giovannucci EL et al (1993) Reproducibility and validity of food intake measurements from a semiquantitative food frequency questionnaire. J Am Diet Assoc 93: 790-796

14. National Diabetes Data Group (1979) Classification and diagnosis of diabetes mellitus and other categories of glucose intolerance. Diabetes 28:1039-1057

15. The Expert Committee on the Diagnosis and Classification of Diabetes Mellitus (1997) Report of the Expert Committee on the Diagnosis and Classification of Diabetes Mellitus. Diabetes Care 20: 1183-1197

16. Hu FB, Leitzmann MF, Stampfer MJ, Colditz GA, Willett WC, Rimm EB (2001) Physical activity and television watching in relation to risk for type 2 diabetes mellitus in men. Arch Intern Med 161:1542-1548

17. Manson JE, Rimm EB, Stampfer MJ et al (1991) Physical activity and incidence of non-insulin-dependent diabetes mellitus in women. Lancet 338:774-778

18. Chiuve SE, Fung TT, Rimm EB et al (2012) Alternative dietary indices both strongly predict risk of chronic disease. J Nutr 142: $1009-1018$
19. Lopez-Garcia E, van Dam RM, Rajpathak S, Willett WC, Manson JE, Hu FB (2006) Changes in caffeine intake and long-term weight change in men and women. Am J Clin Nutr 83:674-680

20. DerSimonian R, Laird N (1986) Meta-analysis in clinical trials. Control Clin Trials 7:177-188

21. Bhupathiraju SN, Pan A, Malik VS et al (2013) Caffeinated and caffeine-free beverages and risk of type 2 diabetes. Am J Clin Nutr 97:155-166

22. Ohnaka K, Ikeda M, Maki T et al (2012) Effects of 16-week consumption of caffeinated and decaffeinated instant coffee on glucose metabolism in a randomized controlled trial. J Nutr Metab 2012: 207426

23. Wedick NM, Brennan AM, Sun Q, Hu FB, Mantzoros CS, van Dam RM (2011) Effects of caffeinated and decaffeinated coffee on biological risk factors for type 2 diabetes: a randomized controlled trial. Nutr J 10:93

24. Yang WS, Wang WY, Fan WY, Deng Q, Wang X (2013) Tea consumption and risk of type 2 diabetes: a dose-response metaanalysis of cohort studies. Br J Nutr 111(8):1329-1339

25. Lioret $\mathrm{S}$, Touvier M, Balin $\mathrm{M}$ et al (2011) Characteristics of energy under-reporting in children and adolescents. Br J Nutr 105:16711680 